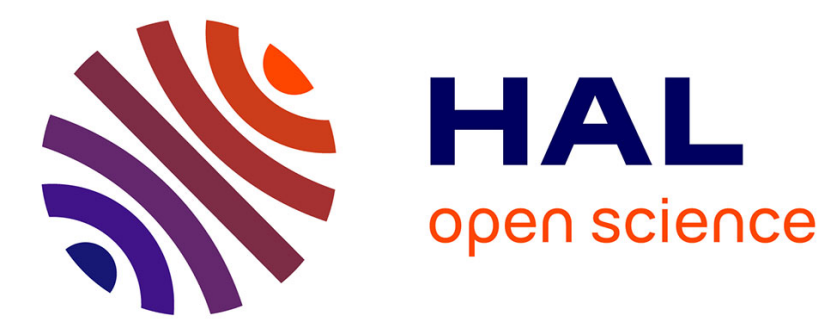

\title{
Petite archéologie de la notion d'ambiance Jean-Paul Thibaud
}

\section{To cite this version:}

Jean-Paul Thibaud. Petite archéologie de la notion d'ambiance. Communications, 2012, Les bruits de la ville, 90, pp.155-174. 10.3406/comm.2012.2659 . hal-00978345

\section{HAL Id: hal-00978345 \\ https://hal.science/hal-00978345}

Submitted on 10 Jul 2017

HAL is a multi-disciplinary open access archive for the deposit and dissemination of scientific research documents, whether they are published or not. The documents may come from teaching and research institutions in France or abroad, or from public or private research centers.
L'archive ouverte pluridisciplinaire HAL, est destinée au dépôt et à la diffusion de documents scientifiques de niveau recherche, publiés ou non, émanant des établissements d'enseignement et de recherche français ou étrangers, des laboratoires publics ou privés.

\section{(이) $\$$}

Distributed under a Creative Commons Attribution - NonCommercial - NoDerivatives| 4.0 


\section{PETITE ARCHEOLOGIE DE LA NOTION D’AMBIANCE}

A l'heure où le domaine des sens entre en force dans la pensée des sciences humaines et sociales, la notion d'ambiance fait figure de proue et prend véritablement toute son ampleur. Sans doute n'est-ce pas un hasard puisque l'ambiance constitue la basse continue du monde sensible, la toile de fond à partir de laquelle s'actualisent nos perceptions et nos sensations. Paradoxalement, alors que nombre de recherches se réclament explicitement de la thématique des ambiances, peu de travaux ont été menés jusqu'alors pour identifier les sources premières et les racines profondes d'une telle notion. Une telle entreprise s'avère pourtant indispensable sitôt que l'on prend celle-ci au sérieux, que l'on se refuse à en faire un mot-valise et que l'on voit en elle une occasion salutaire de renouveler les savoirs sur l'écologie sensible de la ville.

Il s'agit ici de présenter trois perspectives au fondement même de la notion d'ambiance, révélant ainsi l'étendue et la diversité des questions qu'elle permet d'aborder. L'objectif n'est pas tant d'arriver à une définition formelle de cette notion que de mettre en évidence les types de problématique auxquelles elle se prête et le potentiel heuristique qu'elle recèle. Seront explorées une première approche issue de la sémantique historique, une seconde relevant de la psychopathologie existentielle et une troisième portée par l'esthétique phénoménologique.

\section{Sémantique des ambiances}

Au cours des années quarante et cinquante du siècle dernier, plusieurs études linguistiques ont tenté de mettre à jour la sémantique du terme ambiance. Deux démarches principales ont été utilisées à cet égard: une approche étymologique qui vise à identifier l'origine, les conditions d'émergence et les racines possibles du mot «ambiance», et une approche comparative qui s'intéresse aux traductions et aux écarts sémantiques de ce mot dans d'autres langues.

Si l'adjectif ambiant apparaît dès le XVIème siècle dans le milieu scientifique et technique, ce n'est que trois siècles plus tard, aux environs de 1890, que le substantif ambiance commence à être employé en français : en 1885 dans un des Nouveaux contes cruels de Villiers de l'Isle-Adam intitulé «L'amour sublime », en 1890 dans le Traité du Narcisse d'André Gide. Souvent attribué aux frères Goncourt, le substantif ambiance apparaît aussi en 1891 dans un passage de leur Journal. Le milieu littéraire des symbolistes et des impressionnistes constitue ainsi le berceau de ce terme. A cette époque, l'école symboliste fait un grand usage du suffixe - ance qui permet de former entre autres le mot ambiance. Pour Paul Adam, un des représentants de ce mouvement, "ance marque particulièrement une atténuation du sens primitif, qui devient alors moins déterminé, plus vague, et se nuance d'un recul $»^{1}$. Dans son étude inaugurale du mot ambiance, Michaëlsson reprendra à son compte un tel argument pour tenter de montrer que ce néologisme s'inscrit à l'encontre de la raison cartésienne dominante dans la langue française : «Si le français garde toujours intacts des traits fondamentaux qui relèvent de l'ancienne clarté, dans son sens classique, on y rencontre également une part plus large faite à l'intuitif, à l'expressivité, aux demi-jours, aux termes qui suggèrent plus qu'ils ne signifient, aux mots vagues sans contours précis. Parmi ces termes suggestifs et imprécis, le mot ambiance tient une place de premier plan, par son emploi et sa signification, non moins que par la vogue dont il jouit ${ }^{2} \gg$. Si séduisante que soit cette idée, elle ne fait pas l'unanimité des linguistes. Il faudra attendre les travaux de Leo Spitzer pour saisir 
combien cet argument relève d'une simplification un peu hâtive. En effet, si le terme ambiance s'est très rapidement répandu dans le langage courant, dans une acception très large et très ouverte, l'adjectif ambiant a été l'objet de nombreux emplois techniques et scientifiques beaucoup plus précis et rigoureux.

Dans un remarquable essai datant de $1942^{3}$, Leo Spitzer déploie une véritable théorie de la connaissance à partir du terme ambiance. S'il rend hommage aux travaux précurseurs de Michaëlsson, il les dépasse à bien des égards en s'appuyant sur la méthode de la sémantique historique dont il est le fondateur. Comme il le remarque lui-même, le simple usage de dictionnaires pour rendre compte de l'origine et de l'évolution d'un terme ne suffit pas. Ce support d'informations ne donne accès qu'à des «sédiments pétrifiés », et ceux-ci ne constituent que la surface visible des lignes de force et des tensions animant le mot en question. La démarche de Spitzer est bien plus ambitieuse : il s'agit de procéder à une histoire des idées en partant de l'antiquité grecque et latine pour arriver jusqu'à l'époque contemporaine. En opérant de la sorte, Spitzer montre en quoi le mot ambiance s'inscrit dans différentes conceptions du monde. C'est en revenant à ces visions du monde que l'on peut comprendre comment le terme ambiance se dote progressivement d'une épaisseur sémantique, philosophique et scientifique.

Il est hors de propos de synthétiser ici les résultats auxquels aboutit Spitzer dans son essai de sémantique historique, on peut néanmoins en retenir quelques idées centrales. L'argument principal est que le terme ambiance est en relation étroite avec le terme milieu, si bien qu'on ne peut mettre à jour le premier sans s'intéresser en même temps au second. Si ces deux mots s'éclairent mutuellement, c'est à la fois parce qu'ils renvoient tous deux à « ce qui environne les hommes ou les choses » et parce qu'ils sont souvent utilisés conjointement (on parle alors du milieu ambiant). Autrement dit, l'analyse des diverses acceptions et usages du mot milieu donne des indications précieuses sur l'histoire des idées dans laquelle s'inscrit le mot ambiance. Dans de longs développements d'une grande érudition, Spitzer s'attache à identifier les déplacements, évolutions et enjeux de la notion d'ambiance au cours de l'histoire de la pensée occidentale.

S'il est désormais admis que le mot ambiance dérive du verbe latin ambire, Spitzer montre qu'à l'origine le préfixe $a m b$ - ne signifiait pas «autour » ou «ce qui entoure » mais plutôt « des deux côtés » (droite et gauche). Cette remarque est loin d'être anodine, elle permet en effet de mettre en évidence la connotation de protection associée au verbe ambire. Celui-ci renvoyait alors au mouvement des deux bras lors d'une étreinte chaleureuse. Cette idée de protection et de bienveillance, de milieu protecteur en sympathie avec l'homme, ne fait que prolonger la vision du monde des grecs antiques, elle sera toutefois remise en cause avec l'avènement de la science moderne qui cherchera des facteurs déterminants extérieurs à l'homme. Un tournant se produit en particulier avec la conception du milieu ambiant (ambient medium) développée par Newton. Au caractère protecteur et bienveillant de l'ambiance se substitue l'idée d'un milieu régi par un ensemble de lois où l'homme n'est plus la mesure de toutes choses. On passe d'une conception «chaude» soucieuse de la relation charnelle et bienveillante de l'homme à son milieu, à une abstraction «froide » et déterministe faisant de l'homme une entité isolée traversée par un ensemble de forces qui lui échappent ${ }^{4}$.

Malgré cette rupture, on retrouve la conception partagée d'un environnement actif, ayant des effets sur le corps, l'esprit ou le comportement des individus. Cette influence se traduit de différentes manières selon les époques : dans la pensée d'Hippocrate pour qui le climat ou l'atmosphère agit sur la constitution humaine, dans l'Esprit des Lois de Montesquieu, dans la notion de milieu ambiant chez Newton ou encore dans la théorie du milieu de Taine. Bref, si 
la notion d'ambiance est sous-tendue par l'idée d'une force active s'exerçant sur l'être humain, cette force s'est dotée d'une connotation protectrice ou menaçante, en symbiose avec l'homme ou au contraire extérieure à lui, selon les diverses conceptions du monde mises en jeu.

Outre la recherche étymologique, d'autres méthodes ont été utilisées pour tenter de clarifier le terme «ambiance »: rechercher l'équivalent dans d'autres langues, travailler sur l'écart sémantique de mots voisins en français (milieu, climat, atmosphère, environnement) ou rapporter le terme ambiance aux qualificatifs auxquels il se prête dans le langage courant. De tels procédés là encore remettent en cause l'usage courant de dictionnaires : "Le sens précis d'un mot, souvent, ne se laisse pas exprimer dans une simple définition lexicographique : son usage et ses valeurs, en fin de compte, n'apparaissent pleinement qu'à la lumière des exemples et des contextes - ces derniers constituant en somme l'ambiance même des mots, indispensable à la compréhension exacte de leur caractère ${ }^{5}$. » Sans entrer dans le détail, retenons simplement quelques arguments.

Premièrement, la parenté sémantique entre l'ambiance, le milieu ou le climat vient du fait que tous trois renvoient à ce qui nous entoure, nous enveloppe et nous influence. En revanche, la spécificité de l'ambiance par rapport aux deux autres termes est d'insister directement sur la dimension affective : «Milieu, c'est un terme sobre, neutre, strict, qui se ressent toujours, plus ou moins, de sa tradition scientifique. Ambiance et climat sont des termes colorés, évocateurs - climat grâce à la métaphore toujours présente à l'esprit, et ambiance à cause des sentiments qu'il suggère ${ }^{6}$. » Si le climat se définit comme «l'ensemble des conditions atmosphériques auxquelles une région est soumise ", il ne se dote d'une connotation affective que par un usage métaphorique et dans un sens figuré. L'ambiance, au contraire, contient toujours et immédiatement ce caractère «moral» ou affectif. Deuxièmement, contrairement au milieu ou au climat, le terme ambiance est souvent connoté positivement et associé à un lieu ou une situation agréable. Comme le remarque Nilsson-Ehle, si le mot «ambiance » peut être un argument publicitaire vantant les qualités d'un hôtel ou d'un restaurant, «on n'imagine pas l'annonce d'hôtel qui nous dirait *Milieu! Cuisine soignée!, ni naturellement *Climat! Divertissements !, etc. » Troisièmement, alors que les adjectifs accolés à milieu expriment une relation, ceux associés à ambiance indiquent plutôt une qualité. Par exemple, quand on parle de «milieu artistique », on signifie simplement le «milieu des artistes » en distinguant un groupe particulier à l'intérieur de la société. Par contre, quand on parle d'une «ambiance artistique », on sous-entend " un intérieur arrangé avec un goût artistique, contenant de beaux objets d'art, donnant l'impression charmante d'un raffinement de formes et de couleurs ${ }^{7}$. Bref, l'ambiance implique fondamentalement une pensée du qualitatif.

De la sémantique des ambiances, retenons deux leçons. D'une part, si la signification du terme ambiance a évolué au cours du temps, c'est parce que les visions du monde ont ellesmêmes évolué. Loin d'être un terme neutre dépourvu d'enjeux théoriques, l'ambiance s'est dotée progressivement d'une épaisseur sémantique qui engage diverses conceptions de la science. Si l'ambiance devient actuellement un domaine de recherche, elle questionne en retour les paradigmes scientifiques sur lesquels nous nous appuyons pour l'étudier. D'autre part, le terme ambiance ne prend de sens que relativement aux jeux de langage dans lesquels il s'inscrit. Plutôt que de postuler la possibilité d'une définition formelle et univoque, il convient plutôt de mettre en évidence les divers types de discours qu'elle suscite.

\section{Psychopathologie des ambiances}

Dans la première moitié du XXème siècle, un certain nombre de psychiatres de langue allemande reprennent à leur compte les apports de la phénoménologie et jettent les bases de la 
psychopathologie existentielle. Pour aussi différents qu'ils soient, les travaux de Ludwig Binswanger, Erwin Straus ou Eugene Minkowski ${ }^{8}$ opèrent une critique radicale de la psychologie behaviouriste et de l'idéologie objectiviste de l'époque en proposant une nouvelle manière de penser l'être au monde. En s'appuyant souvent sur des cas cliniques et sur leur pratique de thérapeute, ces auteurs entretiennent un dialogue fructueux et sans concession avec la philosophie husserlienne, l'analytique existentiale heideggérienne et la psychanalyse freudienne. Si l'objectif premier est bien de progresser dans la compréhension des désordres mentaux, c'est au prix d'une réflexion fondamentale sur le monde de la vie. Dans le plus connu de ses essais, Binswanger ${ }^{9}$ reprend à son compte et met en exergue la proposition de Kierkegaard : "Il convient plutôt de s'attacher à ce que signifie : être un homme ».

S'il s'agit bien ici de psychopathologie, encore faut-il reconnaître à cette démarche sa véritable portée anthropologique ${ }^{10}$. Ne se satisfaisant pas de la distinction entre le psychique et le somatique et remettant en cause les explications strictement fonctionnelles ou neurophysiologiques, la psychopathologie existentielle se propose de questionner les formes et les structures de l'existence humaine. Ainsi, l'étude de cas cliniques n'est pas close sur elle-même, elle doit plutôt s'ouvrir sur les différentes manières d'être au monde, qu'elles soient «normales» ou «pathologiques ». Autrement dit, les pathologies mentales - et en particulier les psychoses - constitueraient des analyseurs particulièrement pertinents pour rendre compte des modes d'exister.

Deux arguments centraux viennent asseoir cette idée. D'une part, la description attentive et minutieuse de cas cliniques précis ne doit pas se limiter à rendre compte d'une expérience singulière mais doit aussi permettre de dévoiler les traits invariants sur lesquels elle repose. Comme le remarque Minkowski : "Aussi importe-t-il de réserver à la phénoménologie du fait psychopathologique une autonomie entière par rapport et à la clinique et à la psychologie au sens habituel du terme. Ce n'est pas tant à l'individuel en tant que cas singulier que nous opposons le commun et le général, qu'à l'individuel en ce qu'il a de contingent, de variable et d'inconstant nous opposons l'essentiel qui le dépasse et le porte en même temps. ${ }^{11} \gg$ Bref, il s'agit de se détacher du subjectif pour découvrir la structure sous-jacente d'une manière d'être au monde. D'autre part, cette anthropologie ne se focalise pas sur le sujet lui-même mais plutôt sur la morphologie du monde dans lequel il s'inscrit. Autrement dit, c'est le rapport de l'homme au monde, le comment de l'être dans le monde, qui constitue le fil conducteur de l'analyse : "Pas d'existence qui ne soit existence dans le monde ou exister n'est autre chose qu'être dans le monde. De ce fait : $1^{\circ} 1^{\prime}$ 'opposition entre sujet et objet, à laquelle la pensée est venue toujours à nouveau se heurter, est surmontée : le sujet n'existe que dans la mesure où il est dans le monde ; $2^{\circ}$ la psychologie centrée sur un sujet détaché du monde fait place à l'anthropologie, c'est-à-dire à l'étude de l'être humain rattaché par essence au monde dans lequel il est. ${ }^{12}{ }^{\prime}$

C'est sur la base de cette anthropologie clinique que la thématique des ambiances émerge et prend tout son sens. Notons tout d'abord que la notion d'ambiance permet d'identifier certains traits fondamentaux de l'existence humaine. Mais encore, plus que l'ambiance ellemême, c'est l'attitude à l'égard de l'ambiance qui est ici questionnée. Ainsi, à la suite de Bleuler, Minkowski distingue deux principes vitaux qui règlent notre équilibre mental : la syntonie et la schizoïdie ${ }^{13}$. La syntonie désigne le principe qui nous permet de «vibrer à l'unisson avec l'ambiance ». Elle assure le contact vital avec la réalité et renvoie au sentiment d'harmonie avec le monde. La schizoïdie, au contraire, désigne la faculté de «nous détacher de cette même ambiance ». Elle procède de l'élan personnel, permet d'affirmer le moi, de donner sens et direction à l'avenir. Il s'agit là de deux fonctions complémentaires de la vie humaine. Ce n'est que quand une des deux fonctions s'hypertrophie et prend le dessus sur 
l'autre qu'apparaissent des troubles pathologiques : un excès de schizoïdie serait propice au développement d'une schizophrénie, alors qu'un excès de syntonie conduirait plutôt à un trouble maniaco-dépressif.

Partant d'une approche plus délibérément heideggérienne, Binswanger décrit lui aussi des cas cliniques qui conduisent à des conclusions sensiblement identiques. Ainsi, Le cas Susanne $U r b a n^{14}$ fait état d'une patiente qui, suite à un événement traumatique, perd sa capacité de mise à distance et d'articulation du monde dans lequel elle se trouve. En contact immédiat avec l'ambiance, sans possibilité d'éloignement ou de retrait, cette patiente est plongée dans une atmosphère homogène qu'elle ressent comme effrayante. Par cette " atmosphérisation » ${ }^{15}$ du monde, elle se sent dépossédée d'elle-même et sombre alors dans un délire où tout devient menaçant et inquiétant. Dans le cas de la psychose maniaco-dépressive, il en va tout autrement ${ }^{16}$. Quels que soient les événements auxquels il est confronté, rien ne semble atteindre ou toucher le malade. Celui-ci fait montre de sautes d'humeur ou d'idées, passe du coq à l'âne comme si rien n'avait vraiment d'importance. Le monde perd alors de son relief et de sa profondeur, le contact avec l'ambiance semble rompu. Ainsi, pour Minkowski comme pour Binswanger, nos manières d'être au monde semblent indissociablement liées aux types de rapport que nous entretenons avec l'ambiance.

Pour comprendre la façon dont la psychopathologie a thématisé la notion d'ambiance, il faut sans doute revenir aux distinctions opérées entre diverses formes d'espace. Que l'on se réfère à la distinction entre l'《espace orienté » et l' «espace thymique » proposée par Binswanger ${ }^{17}$, à l' « espace clair » et $l^{\prime}$ ' espace noir » chez Minkowski ${ }^{18}$ ou bien encore à l' «espace géographique » et l' «espace du paysage» chez Straus ${ }^{19}$, c'est à partir de la question spatiale qu'un éclairage peut être donné de la notion d'ambiance. Si ces distinctions ne sont pas complètement équivalentes les unes aux autres, elles possèdent néanmoins d'étroites affinités et s'attachent toutes à mettre en évidence ce que l'on pourrait appeler l' «espace ambiant». D'un côté donc, l'espace pragmatique et finalisé, celui de nos actions et perceptions, celui des objets et des pratiques qui s'y rapportent. Les espaces « orienté », «clair » et «géographique » constituent autant de manières de désigner l'espace fonctionnel de la vie quotidienne. De l'autre côté, l'espace affectif et qualifié, celui de nos humeurs et de nos sensations, celui des atmosphères qui nous enveloppent et des sentiments qui nous traversent. Les espaces «thymique », «noir» et du «paysage » se rapportent ainsi au monde ambiant et à nos manières d'être au monde. Si la première façon de vivre l'espace est celle qui est habituellement mise en avant et étudiée d'un point de vue scientifique, la seconde n'est généralement pas prise en compte, ni même thématisée. Pourtant, il en va de la physionomie du monde dans lequel nous vivons, de son caractère plus ou moins hospitalier ou familier, de la communication immédiate que nous avons avec les phénomènes environnants. La distinction entre ces deux formes de spatialité ne doit pas nous induire en erreur. Celles-ci sont toujours simultanément présentes dans l'expérience de tous les jours. Le monde est vécu à la fois comme un monde d'objets à partir desquels nous agissons et nous nous orientons et comme un monde de qualités avec lesquelles nous vibrons et nous nous accordons.

Précisons plus avant ce qu'il en est du monde ambiant. Si nous avons mis l'accent jusqu'à présent sur son versant spatial, encore faut-il ne pas se méprendre sur ce qu'il recouvre. Ce qui est visé ici, ce n'est pas le monde de la représentation mais celui de la présence, non pas le quoi du monde environnant mais le comment de notre être au monde. Autrement dit, et là réside tout l'intérêt et toute la difficulté d'une telle entreprise, il s'agit de saisir ce qui échappe à toute objectivation et thématisation, ce qui relève de l'expérience anté-prédicative du monde $^{20}$. Comme je l'ai déjà mentionné, l'idée d'espace ambiant se rapporte aux structures 
anthropologiques de l'être au monde et ne se réduit en aucun cas à une simple position topologique.

Si les œuvres de Minkowski, Binswanger et Straus s'accordent sur de nombreux points en matière d'ambiance, elles n'empruntent pas pour autant le même chemin de pensée pour saisir un tel domaine. Une des façons de différencier ces divers auteurs est de dégager le rôle que joue la sensorialité dans chacune de ces démarches. Ayant passé une partie de sa vie en France, Minkowski est celui des trois penseurs qui fera un usage explicite du terme « ambiance » lui-même. La notion centrale de contact vital avec la réalité indique que c'est moins l'ambiance elle-même qui importe que l'interaction qu'entretient l'individu avec l'ambiance. Le terme "vital» est ici fondamental dans la mesure où au-delà du contact sensoriel avec l'ambiance, c'est la dynamique de ces contacts qui est visée. C'est ainsi que l'ambiance est à penser en termes de devenir : «L'ambiance ici ne doit point être assimilée à ce que, lorsque nous parlons de perceptions, nous appelons 'monde extérieur', ni par conséquent décomposée en ses prétendus éléments. Elle doit être prise comme un tout vaste et vivant, dans tout son dynamisme primitif, dans lequel par la suite seulement l'homme, à l'aide de procédés analytiques à sa portée, arrive à discerner des êtres vivants, des objets et jusqu'à des excitants physiologiques. L'ambiance, dans ses particularités, n'est point chose faite; elle se fait au contact de l'homme comme celui-ci se fait au sien. L'ambiance primitivement est un océan mouvant. Elle est le devenir ${ }^{21}$. » Cette façon de penser n'exclut pas le monde des sens de l'ambiance. Bien au contraire, il s'agit plutôt de dégager la portée vitale de nos sens en montrant comment chacun d'eux procède d'un mouvement d'ouverture au monde spécifique. Ainsi, à chaque sphère sensorielle correspondrait une attitude particulière de l'être humain à l'égard de la vie ambiante : retentir (l'auditif), se répandre (l'olfactif), goûter (le gustatif), toucher (le tactile) ${ }^{22}$. Pour Minkowski, les métaphores expriment le rôle structurant que joue chaque sens dans la contexture générale de la vie. Bref, ce serait à partir du devenir ambiant que s'articulerait le phénoménal au psychique, le monde matériel au monde spirituel.

Pour Binswanger, l'espace thymique repose sur trois «radicaux existentiels» complémentaires et indissociables : la spatio-temporalité, l'affectivité et la corporéité (à ceuxci pourrait d'ailleurs être rajouté le rapport à soi et à autrui ${ }^{23}$ ). Le fondateur de la Daseinanalyse s'attache ainsi à identifier et décrire le complexe dynamique qui articule ces trois dimensions. Partant de l'idée que le sens de notre rapport au monde est déjà inscrit dans le langage, il propose la notion de «direction de sens » (Bedeutungsrichtung) pour en rendre compte $^{24}$. D'une certaine manière, il s'agit de redonner tout son poids et toute son épaisseur aux expressions du langage courant pour dévoiler les formes et structures fondamentales de l'être-au-monde. Prenons l'exemple paradigmatique de la chute : «Lorsque, brutalement déçus, nous 'tombons du ciel', nous tombons effectivement; mais ce n'est ni une chute purement physique, ni une chute qui l'imite (métaphoriquement ou analogiquement) ou qui est en soi dérivée ; plus exactement, l'essence de la déception brutale et de l'effroi consiste en ceci que l'harmonie du monde ambiant et commun, qui jusqu'ici nous portait, reçoit soudain un choc tel qu'elle vacille. En un tel instant, notre existence est effectivement lésée, arrachée à l'appui qu'elle prend sur le monde et rejetée sur elle-même. Jusqu'à ce que nous trouvions à nouveau un nouvel ancrage dans le monde, notre Dasein total sera dans la direction de signification du trébuchement, de l'affaissement et de la chute. Nous appelons forme cette direction générale de signification et contenu l'effroi brutal, par où nous voyons qu'ici les deux ne sont encore qu' $u n^{25}$. » Ainsi, le phénomène de la chute ne peut être réduit à une de ses acceptions, qu'elle soit physique, morale, affective ou spirituelle. La chute relève d'une direction de sens dans la mesure où elle opère l'unité entre une forme de l'espace-temps vécu, un style de mouvement du corps vivant et une tonalité affective dominante. De ce point de 
vue, une direction de sens rend compte d'une manière d'être au monde spécifique ${ }^{26}$. Binswanger accorde une attention toute particulière à la chute mais il n'en dégage pas moins d'autres directions de sens comme l'ascension, l'étroitesse, l'ampleur, l'éloignement. Si l'espace thymique - tel que caractérisé et spécifié par les directions de sens - est un espace chargé de qualités qui convoquent la corporéité et la sensorialité du sujet, il renvoie d'abord et avant tout à la sphère des sentiments vitaux et pas seulement à celle des phénomènes sensoriels. Autrement dit, ce qui est visé dans les directions de sens, c'est la dimension atmosphérique ou climatique de l'être au monde. De même que Minkowski se saisit des sens de manière à dégager leur portée vitale, Binswanger met surtout l'accent sur le caractère vital des tonalités affectives. Là encore, la sensorialité du sujet n'est pas thématisée en soi ou pour elle-même, elle ne prend tout son sens que rapportée à des états émotionnels et des dispositions d'humeur.

Sans doute revient-il à Erwin Straus d'avoir véritablement développé le versant esthésique de notre présence au monde. Partant d'une critique très serrée du behaviourisme, il élabore la notion de «sentir » pour désigner le mode de relation originaire que nous entretenons avec le monde. Si comme Minkowski et Binswanger il s'interroge lui aussi sur le vivant et sur les modalités de l'existence humaine, il accorde une place essentielle à la dimension sensible de l'expérience vécue. Comme le remarque Renaud Barbaras à propos de Straus : «Le vivant n'est pas le sujet constitué du sentir; il se constitue, au contraire, dans le sentir ${ }^{27}{ }^{2}$. Encore faut-il ne pas se méprendre sur ce que Straus entend par sentir. S'il propose ce terme, c'est d'abord pour le distinguer de la notion de sensation conçue comme une excitation localisée et ponctuelle résultant d'un processus purement physiologique. Bien au contraire, le sentir correspond à un mode de communication global et immédiat avec le monde, c'est par lui que le vivant forme une totalité avec le monde. De ce point de vue, il concerne aussi bien le monde animal que le monde humain. De plus, Straus s'attache à distinguer le sentir du percevoir. Alors que le percevoir est déjà un connaître et engage un « moment gnosique », le sentir désigne avant tout un ressentir qui engage un «moment pathique » dépourvu de toute objectivation ou mise à distance thématique. Avec le sentir, il en va d'un rapport empathique et d'un attachement charnel avec le monde. D'ailleurs, pour Straus, le sentir est indissociable d'un «se mouvoir» dans la mesure où le monde sensible ne se réduit pas à des états de choses ou à des qualités indépendantes du sujet mais sollicite au contraire une activité motrice et mobilise des dispositions affectives. Bref, c'est en se dotant d'une physionomie particulière que les choses m'apparaissent et exercent sur moi leur puissance d'affection : "Le sentir est orienté vers les caractères physionomiques de ce qui est effrayant ou effarouchant. (...) L'empathie est le concept le plus large qui englobe à la fois les actes de séparer et de réunir, ceux de fuir ou de suivre, l'effroi ou l'attrait qui inclut donc aussi bien le sympathique et l'antipathique ${ }^{28}$. $\gg$ C'est en mettant l'accent sur le visage dont se dotent les choses et sur le mouvement d'approche qu'elles suscitent que Straus parvient à faire du monde sensible le vecteur principal de notre présence au monde. L'espace du paysage et les mouvements de la danse constituent à cet égard les deux cas paradigmatiques à partir desquels est présenté le moment pathique propre au sentir. Pour finir, si Straus a sans doute surestimé la distinction entre le pathique et le gnosique, il figure parmi ceux qui se sont rapprochés au plus près de la phénoménalité du monde ambiant ${ }^{29}$.

Pour résumer, trois idées principales peuvent être retenues de la psychopathologie des ambiances. Premièrement, le domaine des ambiances renvoie à un niveau vital et nonthématique de l'expérience. En deçà d'un rapport de connaissance, d'objectivation ou de représentation du monde, l'ambiance relève plutôt de la présence au monde. Elle engage à la fois la manière dont nous nous sentons dans le monde et la façon dont nous le ressentons. Pour autant, on ne doit pas creuser outre mesure la distinction entre le sentir et le percevoir 
mais plutôt chercher des moyens d'articuler ces deux versants ${ }^{30}$. Deuxièmement, l'ambiance renvoie d'abord et avant tout à des tonalités affectives. Néanmoins, loin d'être indépendantes d'autres composantes de l'expérience, elles ne prennent de sens que rapportées à des formes spatio-temporelles, des qualités de mouvement et des données sensibles. Troisièmement, l'ambiance peut être déclinée à partir d'une logique modale. Elle ne désigne pas le «quoi» de l'expérience mais le « comment». L'articulation de ses diverses composantes permet ainsi d'identifier des manières d'être particulières qui engagent aussi bien le sujet lui-même que le monde dans lequel il se trouve. Bref, l'ambiance ne serait autre qu'une manière d'identifier, de décrire et de distinguer des « styles d'exister ${ }^{31}$ ».

\section{Esthétique des ambiances}

Après avoir traité de la sémantique et de la psychopathologie des ambiances, qu'en est-il maintenant de l'approche esthétique ? La notion d'ambiance concerne l'esthétique au premier chef dans la mesure où elle pose explicitement la question de la sensorialité humaine. Deux éléments de cadrage positionnent l'esthétique vis-à-vis de la thématique des ambiances. D'une part, l'ambiance convoque une «esthétique environnementale » en aucun cas réductible à une esthétique des beaux-arts. Ce n'est pas l'œuvre d'art qui est au centre de cette esthétique mais bien plutôt la nature, comprise au sens large du terme (en incluant la ville et l'urbain). Pourtant, si cette esthétique procède à la fois d'une pente narrative et d'une pente ambiantale, la première a pour l'instant largement dominé la seconde ${ }^{32}$. D'autre part, l'ambiance permet de revenir au sens premier de l'esthétique, c'est-à-dire conçue comme théorie de la perception sensible. Il s'agit alors de restaurer une pensée de l'aisthesis qui dépasse le jugement de goût, réhabilite la place du corps, de la sensibilité et de l'émotion, relativise le poids de la sémiotique et le primat accordé au langage ${ }^{33}$. L'ambiance conduit ainsi à repenser à la fois l'objet de l'esthétique et la discipline esthétique elle-même.

Limitons-nous ici à l'esthétique des ambiances telle que développée par Jean-François Augoyard et Gernot Böhme ${ }^{34}$. Alors que pour le premier, c'est bien le terme « ambiance » qui est utilisé, pour le second c'est le terme «atmosphère (de langue allemande, Böhme emploie le mot Atmosphäre ${ }^{35}$ ). Si ces deux pensées se sont construites indépendamment l'une de l'autre, il n'en existe pas moins de nombreux points communs. Outre le fait qu'elles cherchent à revenir à une théorie de la perception sensible, elles mettent toutes deux l'accent sur la composante architecturale et plus généralement sur la dimension spatiale et matérielle des ambiances ${ }^{36}$. Il s'agit bien dans les deux cas d'élaborer une esthétique des ambiances appliquée à l'espace construit. Mais encore, l'esthétique des ambiances ne renvoie pas seulement à des architectures monumentales ou à des édifices de référence mais convoque plus largement l'ensemble des situations de la vie quotidienne. S'inscrivant contre une conception muséale de l'art et refusant l'amalgame entre expérience artistique et expérience esthétique, une telle approche donne voix aux situations les plus ordinaires.

L'importance accordée au caractère contextuel des ambiances conduit à proposer une esthétique écologique. Pour Augoyard, l'objectif est de développer des approches in situ et de construire des outils interdisciplinaires qui convoquent aussi bien «les signaux physiques perceptibles, l'ensemble des normes, règles, codes et références, enfin les instrumentations, fonctions et usages affectés à la forme construite ${ }^{37} \gg$. Pour Böhme, le problème consiste à penser le lien entre des qualités environnementales et la sensibilité humaine de manière à mieux comprendre «comment on se sent dans un environnement ${ }^{38} »$. Si les termes en présence pour l'un et l'autre ne sont pas les mêmes, chacun s'accorde à penser la complexité des situations en proposant d'articuler entre elles des composantes contextuelles hétérogènes.

Cette logique de l'articulation se retrouve d'ailleurs à tous les niveaux du questionnement. Tout se passe comme si l'ambiance n'était autre que le lieu à partir duquel diverses 
composantes ou polarités devaient être reliées ou ressaisies dans une même dynamique. D'ailleurs, Böhme fait constamment référence à l'atmosphère comme un " entre-deux » (inbetween). Pour la clarté de l'exposé, je distinguerai trois niveaux d'articulation principaux.

Premièrement, il en va du rapport entre le sujet et l'objet. Comme le remarque Augoyard ${ }^{39}$, on peut revenir à la définition du terme ambiance telle que donnée dans les dictionnaires français : «atmosphère matérielle et morale qui environne un lieu, une personne... ». Pour aussi élémentaire qu'elle soit, cette définition met en évidence la conjonction entre la dimension «matérielle » et «morale ». Loin d'être anodine, cette articulation pose problème et nécessite de développer une attention aux «quasi-êtres » et «semi-choses » conduisant à une esthésique des intermédiaires ${ }^{40}$. De son côté, Böhme insiste sur le fait que l'atmosphère est à la fois objective puisqu'elle émane des choses et qu'elle peut être produite à partir d'arrangements matériels, et subjective puisqu'elle ne peut être définie indépendamment des personnes qui l'éprouvent. Bref, l'atmosphère serait « la manifestation de la co-présence entre sujet et objet ${ }^{41} »$.

Deuxièmement, il en va du rapport entre les sens. Ici, Augoyard distingue l'ambiance (au singulier) des ambiances (au pluriel). Si la science et la technique ont l'habitude de dissocier les diverses modalités sensorielles pour mieux étudier l'environnement, la question reste entière quant à sa capacité à rendre compte des phénomènes intersensoriels ou amodaux : «Alors que l'architecte produit une ambiance, nous lui proposons de mettre bout à bout des ambiances, l'une thermique, l'autre acoustique, etc. Pouvons-nous seulement définir scientifiquement ce qu'est une ambiance architecturale $?^{42} 》$. Dans le même ordre d'idée, Böhme remet en cause la tendance à dissocier les sens dans un premier temps pour chercher à les réunifier par la suite. En reprenant à nouveaux frais la question de la synesthésie, il montre au contraire que l'unité sensible d'une situation préexiste à la différenciation entre les sens. C'est sur la base de l'atmosphère, conçue comme un arrière-plan global et indivis que s'opèrerait la discrimination de détails et la distinction entre telle ou telle modalité sensible ${ }^{43}$.

Troisièmement, il en va du rapport entre la réception et la création. L'ambiance relève à la fois de ce qui peut être perçu et de ce qui peut être produit. Mieux encore, elle tend à questionner une telle distinction dans la mesure où la perception est elle-même action. De même que l'architecte ou le scénographe agence matériellement des formes sensibles, les usagers configurent par leurs actes le milieu dans lequel ils se trouvent. Augoyard avance ainsi l'hypothèse que « notre relation avec l'environnement sensible et formel est à concevoir comme un échange, une circulation constructive entre le donné et le configuré, le senti et l'agi, le perceptible et le représentable ${ }^{44} \gg$. Böhme le dit lui aussi à sa manière quand il indique que la production d'une atmosphère ne procède pas simplement de la conception d'un objet mais toujours en même temps de «la création des conditions de son apparence ${ }^{45} »$. De ce point de vue, la manière dont le monde apparaît engage aussi bien le travail du concepteur que l'activité de l'habitant.

S'il existe une grande affinité entre ces deux auteurs - en particulier dans leur recours à la phénoménologie -, relevons tout de même quelques traits spécifiques à chacun. Notons tout d'abord que la dimension objective et matérielle de l'ambiance n'est pas abordée exactement dans les mêmes termes. Chez Augoyard, elle se rapporte à la fois au signal physique et à l'espace construit alors que chez Böhme elle relève plutôt du monde des choses. Partant de l'idée que tout signal physique incorpore nécessairement les propriétés du cadre bâti dans lequel il se produit, Augoyard propose de développer une physique contextuelle : «Le son situé traverse un espace de propagation qui lui donne une certaine qualité hic et nunc; par exemple : temps de réverbération et timbrage pour le son, réflexion particulière, modification de température, de couleur, organisation des ombres pour la lumière, turbulences particulières 
des flux de l'air autour de certaines configurations architecturales, volatilité variable des odeurs en fonction de la vitesse du vent. Le signal physiquement isolable a posteriori n'existe qu'à travers cette incorporation spatio-temporelle entièrement dépendante des qualités morphologiques et matérielles du lieu ${ }^{46}$. » Une telle perspective renoue avec la « maîtrise des ambiances » telle qu'enseignée dans les écoles d'architecture en réintroduisant la part du contexte spatial dans les procédures métrologiques. L'enjeu ne consiste pas moins à repenser la complémentarité entre le quantitatif et le qualitatif. Mais encore, si le signal physique n'a d'existence que rapporté au cadre bâti, il n'a véritablement de sens qu'à partir du moment où il est perçu et filtré par les codes, normes, représentations et interactions sociales en vigueur dans le lieu. Bref, comme le constate Augoyard, cette chaîne de conformation du signal aux multiples traits contextuels en présence (spatio-temporel, perceptif, culturel, social) «aboutit à la définition d'un objet central qui n'est plus le signal mais le phénomène ${ }^{47} \gg$. Autrement dit, le questionnement ne porte pas tant sur le signal physique lui-même ou sur l'ambiance en général mais bien plutôt sur ce lieu d'articulation que constitue le " phénomène d'ambiance ».

Pour sa part, Böhme aborde la dimension matérielle de l'ambiance à partir du monde des choses. Son argument central consiste à affirmer le caractère «extatique » des choses. Il remet en cause l'ontologie classique de la chose qui considère ses qualités comme des déterminations $^{48}$. Autrement dit, selon cette conception traditionnelle, les qualités d'une chose (forme, couleur, odeur, etc.) constitueraient ce qui la distingue d'une autre. Chaque chose possèderait une unité interne, serait close sur elle-même, distincte des autres et indépendante de son environnement immédiat. Pour Böhme au contraire, les choses ont le pouvoir de se révéler elles-mêmes, d'interagir entre elles et de se diffuser à l'extérieur d'elles-mêmes. Comme l'illustre la peinture, la couleur d'un objet peut être modifiée par celle d'un autre à proximité, la forme d'une chose peut créer des lignes de forces et des suggestions de mouvement. Un des exemples donnés par Böhme pour illustrer son propos mérite d'être présenté : «Si nous disons par exemple : un bol est bleu, alors nous pensons à une chose qui est déterminée par sa couleur bleue, qui la distingue des autres choses. Cette couleur est quelque chose que ce bol 'a'. En plus de cette 'bleuité' (blueness) on peut aussi demander si un tel bol existe. Son existence est déterminée alors par sa localisation dans l'espace et le temps. La 'bleuité' du bol peut pourtant être pensée d'une toute autre façon, à savoir comme la manière, ou mieux, une manière à partir de laquelle le bol est présent dans l'espace et rend sa présence perceptible. La 'bleuité' du bol est alors pensée non pas comme une qualité restreinte au bol et qui adhère à lui, mais au contraire comme quelque chose qui se diffuse vers son environnement, en colorant ou en 'teintant' d'une certaine manière cet environnement, comme Jakob Böhme le dirait ${ }^{49}$. C'est ainsi que chaque chose affecterait son environnement de sa présence.

Mais encore, en mettant en évidence cette dimension extatique des choses, Böhme introduit un troisième terme habituellement négligé dans les théories de la perception : le médium. Comme il le dit lui-même : «La structure de la perception n'est pas 'je vois quelque chose' mais plutôt 'dans le médium, la présence des choses est perceptible" ${ }^{50}$ ". Ici se trouve sans doute le moment clé de la pensée de Böhme en matière d'atmosphère. L'introduction du médium comme troisième terme conduit à un double développement. D'une part, c'est à partir de lui qu'il est possible de dépasser l'idée selon laquelle la perception pourrait être réduite au simple fait d'identifier ou de remarquer des objets. En effet, le médium est le support à partir duquel le monde se dote d'une certaine physionomie et dispose le sujet percevant dans un certain état corporel et affectif. D'autre part, du point de vue de l'expérience, l'atmosphère n'est autre que le médium, ou plus précisément, l'état du médium dans une situation donnée. Autrement dit: "L' 'objet' premier de la perception est l'atmosphère. Ce qui est initialement et immédiatement perçu n'est pas des sensations, ni des 
formes ou des objets ou leurs constellations, comme la psychologie de la Gestalt le pensait, mais des atmosphères, arrière-plan sur la base duquel le regard analytique distingue de telles choses comme des objets, des formes, des couleurs, etc ${ }^{51}$. »

Augoyard et Böhme s'appuient ainsi sur des catégories sensiblement différentes. Pour le premier, c'est l'idée de phénomène d'ambiance qui prévaut, avec ses six entrées complémentaires (signal physique, forme spatio-temporelle, percept, représentation, code et norme, interaction sociale). Pour le second, la problématique de l'atmosphère se construit plutôt autour de la notion de présence, avec ses trois versants principaux (la chose, le médium, les sens).

Pour résumer, trois apports principaux peuvent être retenus de l'esthétique des ambiances. Premièrement, elle permet de penser à nouveaux frais la perception sensible en relevant son caractère éminemment contextuel, en l'inscrivant dans la vie sociale quotidienne, en l'ouvrant à l'ensemble des sens et en redonnant toute son importance aux facteurs physiques et matériels. Autrement dit, l'intérêt porté à la phénoménalité de l'espace construit conduit à développer l'idée d'ambiances architecturales et urbaines. Deuxièmement, une telle esthétique phénoménologique aide à rendre compte de la complexité d'une ambiance en convoquant une démarche modale et interdisciplinaire. De ce point de vue, plutôt que de dissocier les termes d'une ambiance et isoler les facteurs qui la composent, il s'agit de les articuler et de les faire tenir ensemble. Bien que prenant des chemins différents selon les auteurs, le propos est d'établir des liaisons et des continuités qui donnent consistance à une ambiance. Troisièmement, l'esthétique des ambiances ressortit à une approche dynamique. Plutôt que de saisir l'ambiance comme une donnée ou un état, il s'agit de la penser comme un processus en acte relevant autant de l'activité habitante que de celle du concepteur.

\section{La portée de la notion d'ambiance}

La notion d'ambiance est une notion de grande portée heuristique et opératoire, ouverte à une diversité d'approches et d'usages. Elle se dote de tout son sens et de toute sa puissance dès lors qu'elle est mise en œuvre à partir d'une perspective particulière. C'est en la mettant à l'épreuve de questionnements précis et spécifiques que l'on peut le mieux saisir l'étendue de ses conséquences et de ses implications. Outre les trois chemins explorés ici, d'autres auraient pu être empruntés, allant de la psychologie environnementale à l'architecture sensible, de la géographie humaine à la création artistique, de la pédagogie à la dramaturgie. On le voit, une des originalités de la notion d'ambiance est de favoriser la circulation entre les savoirs et l'articulation entre les disciplines. Notion nomade et transverse s'il en est, l'ambiance ouvre une alternative originale au dualisme de l'objet et du sujet, du sensible et de l'intelligible, de l'actif et du passif. Elle participe sans doute de la sensibilisation de la pensée.

Il ne faut pas se tromper : la difficulté à appréhender un tel domaine de pensée ne doit pas conduire à des erreurs fréquentes. C'est ainsi que l'ambiance n'est en aucun cas assimilable à l'environnement. S'il existe bien des affinités entre ces deux termes, l'ambiance s'adosse sur le plan de la sensorialité, des affects et de l'expérience vécue et ne peut pas se conformer à une démarche par trop objectiviste ou positiviste. Il en va ici des implications épistémologiques de la notion d'ambiance. Par ailleurs, l'ambiance n'est pas non plus d'ordre purement subjectif. Nous avons vu qu'elle ne peut se passer de la matérialité de l'espace construit et aménagé (on parle alors d'ambiances architecturales et urbaines) et qu'elle convoque par ailleurs une dimension anthropologique et collective irréductible à l'expérience individuelle. Enfin, est-il besoin de dire combien l'ambiance bien comprise est aux antipodes d'une notion cosmétique ? Loin d'être un simple surplus de luxe ou de confort, elle aide à penser le versant existentiel de l'expérience humaine. C'est dire si l'ambiance ancre le monde des sens au cœur même de l'habiter et en constitue une condition de possibilité. 


\section{Jean-Paul THIBAUD}

CRESSON / UMR 1563 Ambiances Architecturales et Urbaines

Email : jean-paul.thibaud@grenoble.archi.fr

${ }^{1}$ Cette citation de Paul Adam est donnée par Alexis François : «Suffixe littéraire -ance », Vox Romanica. Vol IV, Bern, Ursprünglicher Erscheinungsort, 1939, p. 20-34. Comme le montre Leo Spitzer, une étude plus attentive de ce suffixe révèle qu'il indique plutôt la perpétuation ou la subsistance d'un état d'être, quelque chose qui perdure et se prolonge dans le temps (Le français moderne, Vol. VII, 1939, p. 276).

${ }^{2}$ Karl Michaëlsson, « Ambiance », Studia Neophilologica, Vol. XII, 1939, p. 91-119.

${ }^{3}$ Leo Spitzer, «Milieu and Ambiance : an Essay in Historical Semantics », Philosophy and Phenomenological Research, Vol. III, 1942, p. 1-42 et p. 169-218.

${ }^{4}$ Notons que certains penseurs, tel Goethe ou les frères Goncourt, n'ont pas manqué de réagir à cette conception moderne du milieu et ont tenté de renouer avec les leçons de l'Antiquité.

${ }^{5}$ Hans Nilsson-Ehle, «Ambiance, Milieu et Climat », Studia Neophilologica, Vol. XXIX, n 2, 1957, p. 181191.

${ }^{6}$ Ibid.

${ }^{7}$ Ibid.

${ }^{8}$ D'autres penseurs de toute première importance - en liens étroits avec l'analyse existentielle - méritent au moins d'être mentionnés, tels Viktor Von Weizsacker, Frederik Jacobus Johannes Buytendijk ou Henri Maldiney pour ne citer que quelques-uns.

${ }^{9}$ Ludwig Binswanger, Le rêve et l'existence, traduction de Jacqueline Verdeaux, introduction de Michel Foucault, Bruxelles, Desclée de Brouwer, 1954.

${ }^{10} \mathrm{C}$ 'est précisément à cette dimension anthropologique que Michel Foucault s'intéresse dans son introduction à Le rêve et l'existence de Binswanger.

${ }^{11}$ Eugène Minkowski, «Phénoménologie et analyse existentielle en psychopathologie », In Ecrits cliniques, Ramonville Saint-Agne, Erès, 2002, p. 95-138

12 Ibid.

${ }^{13}$ Eugène Minkowski, Le temps vécu, Paris, PUF, 1995. Remarquons ici l'affinité étroite entre cette distinction de Minkowski et celle opérée par Gilbert Durand entre le régime nocturne et le régime diurne de l'imaginaire (Les structures anthropologiques de l'imaginaire, Paris, Bordas, 1969).

${ }^{14}$ Ludwig Binswanger, Le cas Suzan Urban. Etude sur la schizophrénie, Paris, Editions Gérard Monfort, 2002.

${ }^{15}$ Sur la notion d' « atmosphèrique » d'un point de vue psychopathologique et sur l'importance du sens oral dans l'atmosphérisation du monde, se reporter à l'ouvrage fondamental de Hubertus Tellenbach, Goût et Atmosphère, Paris, PUF, 1983.

${ }^{16}$ Ludwig Binswanger, Sur la fuite des idées, Grenoble, Jérôme Million, 2000.

${ }^{17}$ Ludwig Binswanger, Le problème de l'espace en psychopathologie, Préface et traduction de Caroline GrosAzorin, Toulouse, Presses Universitaires du Mirail, 1998.

${ }^{18}$ Eugène Minkowski, «Vers une psychopathologie de l'espace vécu », In Le temps vécu, Paris, PUF, 1995, p. 366-398.

${ }^{19}$ Erwin Straus, «Les formes du spatial. Leur signification pour la motricité et la perception », In Jean-François Courtine (dir.), Figures de la Subjectivité, Paris, Editions du CNRS, 1992, p. 15-49.

${ }^{20}$ Notons que l'histoire de la philosophie est traversée par cette préoccupation, qu'il s'agisse de la chôra chez Platon, des synthèses passives chez Husserl ou du jugement réfléchissant chez Kant.

${ }^{21}$ Eugène Minkowski, « Constitution et conflit », In Ecrits cliniques... op.cit., p. 67-79.

22 Eugène Minkowski, Vers une cosmologie, Paris, Payot, 1999.

${ }^{23}$ Je pense en particulier à l'importance qu'il donne au phénomène de la rencontre. 
${ }^{24}$ Pour une mise en perspective récente de cette notion, se reporter à Jeanine Chamond (dir.), Les directions de sens. Phénoménologie et psychopathologie de l'espace vécu, Argenteuil, Le Cercle herméneutique, 2004.

${ }^{25}$ Ludwig Binswanger, Le rêve et... op.cit.

${ }^{26}$ Comme le remarque Henri Maldiney : «Son sens est au-delà ou plutôt en-deçà de toutes ces acceptions locales. Et il doit être appelé une direction de sens, cette alliance de mots rendant sensible l'unité du sensdirection et du sens-signification - qui est celle précisément de l'esquisse existentielle et du dévoilement de l'être qui s'opère en elle » (Regard, Parole, Espace, Paris, L'Age d'Homme, 1973).

27 Renaud Barbaras, «Affectivité et mouvement: le sens du Sentir chez E. Straus », Alter. Revue de Phénoménologie, $\mathrm{n}^{\circ}$ 7, 1999, p. 15-29.

${ }^{28}$ Erwin Straus, Du sens des sens, Grenoble, Jérôme Million, 1989.

29 Comme l'indique Henri Maldiney (op.cit.), la pensée de Straus «commence là où finit l'analyse intentionnelle de Husserl, à cette hylétique qu'il a nommée sans pouvoir l'édifier ». Pour une présentation synthétique de cette question, se reporter à Michèle Gennart, «Une phénoménologie des données hylétiques estelle possible ? », Etudes Phénoménologiques, n 4, 1986, p. 19-46.

${ }^{30}$ Ainsi, Renaud Barbaras (op.cit.) remarque à juste titre : «Il va de soi que nous ne vivons pas exclusivement dans cette dimension pathique dans laquelle l'animal, lui, est enfermé ; nous avons toujours déjà dépassé la stricte relation empathique au profit d'une mise à distance thématique qui saisit les expressions comme déterminations d'une chose, nous vivons dans l'univers de la perception. » Ou bien encore, en formulant un peu autrement cette même idée, Maria Villela-Petit écrit ainsi : «En tant qu'êtres humains nous ne saurions en rester à la seule dimension du sentir, sous peine de ne pas avoir accès à la pensée, mais cette dimension de notre êtreau-monde, nous ne la quittons non plus jamais, sauf dans les cas extrêmes de rupture pathologique de notre communication pathique avec le monde » («Espace, temps, mouvement chez Erwin Straus », In Jean-François Courtine (dir.), Figures... op.cit., p. 51-69).

${ }^{31}$ Henri Maldiney, op.cit.

32 Cheryl Foster distingue l'approche «narrative » qui consiste à lire et écrire l'environnement comme une histoire en s'appuyant sur une sémiotique de l'indexicalité et l'approche «ambiantale » qui insiste sur la sensibilité et résiste à une formulation langagière («The Narrative and the Ambient in Environmental Aesthetics », The Journal of Aesthetics and Art Criticism, Vol. 56, n², 1998, p. 127-137).

33 C'est avec de tels arguments que Gernot Böhme construit son projet d'une «nouvelle esthétique »: « Aesthetics Knowledge of Nature », Issues in Contemporary Culture and Aesthetics, n5, 1997, p. 27-37.

${ }^{34}$ Une esthétique des ambiances se retrouve également chez d'autres auteurs tout à fait importants. Parmi eux, citons en particulier Martin Seel, Yuriko Saito, Pierre Sansot, Henri Maldiney, Herman Schmitz, Michael Hauskeller. Ces perspectives mériteraient de toute évidence un développement en tant que tel.

${ }^{35}$ Gernot Böhme emprunte la notion d' « atmosphère » à la philosophie du corps d'Hermann Schmitz. Tout en reconnaissant cet apport, Böhme opère une critique de la pensée de Schmitz et propose une lecture originale et personnelle de cette notion. Pour Böhme, la pensée de Schmitz reste limitée quant à la construction d'une esthétique des ambiances pour deux raisons principales: Schmitz ne s'émancipe pas complètement d'une conception classique de l'esthétique qui réduit celle-ci au champ artistique ; il ne développe que le versant subjectif de l'atmosphère et ne prend pas suffisamment en compte sa composante matérielle et objective. Voir Gernot Böhme, «Atmosphere as the Fundamental Concept of a New Aesthetics », Thesis Eleven, n³6, 1993, p. 113-126.

${ }^{36}$ Divers numéros de revue d'architecture confirment si besoin est l'inscription des ambiances au sein de la discipline architecturale. Pour mémoire : «Constructing Atmospheres », Daidalos, n 68, 1998 ; « Ambiances architecturales et urbaines », Les Cahiers de la Recherche Architecturale, n 42/43, 1998 ; « Atmosphère », Faces. Journal d'architecture, ${ }^{\circ} 67,2010$.

37 Jean-François Augoyard, «Eléments pour une théorie des ambiances architecturales et urbaines », Les Cahiers de la Recherche Architecturale, ${ }^{\circ}$ 42/43, 1998, pp. 13-23

${ }^{38}$ Gernot Böhme, «Acoustic Atmospheres. A Contribution to the Study of Ecological Aesthetics », Soundscape. The Journal of Acoustic Ecology, Vol. I, n ${ }^{\circ}$, 2000, p.14-18.

${ }^{39}$ Jean-François Augoyard, « Eléments pour une théorie... op.cit. 
40 Jean-François Augoyard, «Faire une ambiance?» In Jean-François Augoyard (dir.), Faire une ambiance, Bernin, A la Croisée, 2011, p. 17-35

${ }^{41}$ Gernot Böhme, «Atmosphere as An Aesthetic Concept », Daidalos, n42/43, 1998, p. 112-115.

42 Jean-François Augoyard, «Eléments pour une théorie... op.cit.

${ }^{43}$ Gernot Böhme, « Über Synästhesien / On Synaesthesiae », Daidalos, n 15, 1991, p. 26-37.

44 Jean-François Augoyard, «Eléments pour une théorie... op.cit.

45 «It is never purely a question of designing an object but always, at the same time, of creating the conditions for its appearence », Gernot Böhme, « On Synaesthesiae... op.cit.

46 Jean-François Augoyard, «L'environnement sensible et les ambiances architecturales », L'espace Géographique, $\mathrm{n}^{\circ} 4$, 1995, p. 302-318.

47 Ibid.

${ }^{48}$ Le développement qui suit s'appuie en particulier sur l'article suivant : Gernot Böhme, G. « Atmosphere as the Fundamental... op.cit.

${ }^{49}$ Ibid. (traduction personnelle de l'anglais).

${ }^{50}$ Gernot Böhme, «An Aesthetic Theory of Nature : An Interim Report », Thesis Eleven, n³2, 1992, p. 90102.

${ }^{51}$ Gernot Böhme, «Atmosphere as the Fundamental...» op.cit. (traduction personnelle de l'anglais).

\section{Résumé.}

A l'heure où le domaine des sens entre en force dans la pensée des sciences humaines et sociales, la notion d'ambiance fait figure de proue et prend véritablement toute son ampleur. Paradoxalement, alors que nombre de recherches se réclament explicitement de la thématique des ambiances, peu de travaux ont été menés jusqu'alors pour identifier ses sources premières et ses racines profondes. Cet article présente trois perspectives au fondement même de la notion d'ambiance: la sémantique historique, la psychopathologie existentielle et l'esthétique phénoménologique. L'objectif est de mettre en évidence les enjeux de la notion d'ambiance et le potentiel heuristique qu'elle recèle. Il est montré comment l'ambiance permet d'ancrer le monde des sens au cœur de l'expérience humaine et de l'habiter.

Mots-clés : ambiance, sensible, sémantique, psychopathologie, esthétique

\section{Abstract.}

The domain of the senses has developed apace in social sciences and the notion of ambiance is becoming increasingly present in this new field of research. Although numerous bodies of work deal explicitly with ambiances, very few of them identify the primary sources and major roots of this notion. This paper presents three perspectives which establish the notion of ambiance: historical semantic, existential psychopathology and phenomenological aesthetics. The aim of this paper is to point out its issues and its potential. This article shows how the notion of ambiance helps to introduce the world of the senses in human experience and in dwelling spaces.

Key words : ambiance, senses, semantic, psychopathology, aesthetics 